\title{
Fungal infection of the colon
}

This article was published in the following Dove Press journal:

Clinical and Experimental Gastroenterology

21 October 2014

Number of times this article has been viewed

\section{Surat Praneenararat \\ Division of Gastroenterology, \\ Department of Medicine, Prince of Songkla University, Songkhla, Thailand}

Abstract: Fungi are pathogens that commonly infect immunocompromised patients and can affect any organs of the body, including the colon. However, the literature provides limited details on colonic infections caused by fungi. This article is an intensive review of information available on the fungi that can cause colon infections. It uses a comparative style so that its conclusions may be accessible for clinical application.

Keywords: fungus, colitis, large bowel, large intestine

\section{Introduction}

Fungi are pathogens that commonly infect immunocompromised patients. At present, the incidence of these pathogens in disease causation is gradually increasing as a result of increased use of immunosuppressive drugs, chemotherapy, and transplantation as well as infections with the human immunodeficiency virus. Fungal infections can affect any organ, including the colon. ${ }^{1-3}$ Nevertheless, only limited details of colonic infection caused by fungi are available in the literature. ${ }^{4,5}$ This article aims to provide an intensive review of research on fungal infection of the colon in a concise, comparative style for easy clinical application.

\section{Methods}

The author initiated the review by researching fungi that can cause colonic infection on MEDLINE, in major textbooks, and existing research literature that review fungal infections of the colon. ${ }^{4-7}$ In MEDLINE, the author used keywords from two groups. The first group consisted of names of the fungi or infections related to the fungi, including "aspergillosis", "Aspergillus", "Blastomyces", "blastomycosis", "Candida", "candidiasis", "chromoblastomycosis", "Cladophialophora", "Coccidioides", "coccidioidomycosis", "cryptococcosis", "Cryptococcus", "dermatophyte", "dermatophytosis", "Epidermophyton", "eumycetoma”, "Fonsecaea", "fusariosis", "Fusarium", "Histoplasma", "histoplasmosis", "Madurella", "Microsporum", "mucormycosis", "Paracoccidioides", "paracoccidioidomycosis", "penicilliosis", "Penicillium", "phaeohyphomycosis", "Pneumocystis", "pneumocystosis", "scedosporiosis", "Scedosporium", "Sporothrix", "sporotrichosis", "Trichophyton", "Trichosporon", "trichosporonosis", "Zygomycetes", and "zygomycosis". The second group of keywords consisted of words related to locations of the disease under our focus, including "colitis", "colon", "colonic", "enterocolitis", "large bowel", "large intestinal", and "large intestine". Finally, it was determined that infections of the colon that have been reported to be caused by fungi were aspergillosis, candidiasis, cryptococcosis,
Correspondence: Surat Praneenararat Division of Gastroenterology,

Department of Medicine,

Prince of Songkla University, I5 Kanjanavanich Road, Hat-Yai, Songkhla, Thailand, 901 10

Tel +668044I 0174

Email goodycherry@hotmail.com
Clinical and Experimental Gastroenterology 2014:7 4I5-426

Dovepress (c) (7) (8) 2014 Praneenararat. This work is published by Dove Medical Press Limited, and licensed under Creative Commons Attribution - Non Commercial (unported, v3.0) permission from Dove Medical Press Limited, provided the work is properly attributed. Permissions beyond the scope of the License are administered by Dove Medical Press Limited. Information on how to request permission may be found at: http://www.dovepress.com/permissions.php 
histoplasmosis, paracoccidioidomycosis, penicilliosis, pneumocystosis, scedosporiosis, and zygomycosis. Details of each fungus were further reviewed from articles retrieved on MEDLINE using the keywords as described above, citations to these articles, and references in major textbooks. In MEDLINE, all types of articles, including reviews, case series, case reports, editorials, and letters, up to June 30, 2014 were included. Initially, 124 articles were found. Only articles which were in English or had an English-language abstract with complete necessary detail that had a definite diagnosis of fungal infection of the colon, including direct visualization of typical fungus or positive fungal culture in colonic specimens, were selected. Cases of candidiasis, which is considered a normal commensal of the human gastrointestinal tract, also needed to have one of the following criteria as per the accepted case reports and revised European Organization for Research and Treatment of Cancer/Invasive Fungal Infections Cooperative Group and National Institute of Allergy and Infectious Diseases Mycoses Study Group criteria: deep tissue invasion in the colon, or positive Candida spp. in superficial mucosa with positive blood culture, or positive Candida spp. in superficial mucosa with evidence in response to treatment. ${ }^{8-14}$ Candida spp. in only superficial mucosa without positive blood culture or evidence in response to treatment were excluded. Dissemination was defined as involvement of two or more noncontiguous organs. ${ }^{15-19}$

At present, histoplasmosis, ${ }^{20,21}$ candidiasis, ${ }^{13}$ and zygomy$\operatorname{cosis}^{22-25}$ have already been reviewed. In cases of other fungal infections, including cryptococcosis, ${ }^{26-35}$ penicilliosis, ${ }^{36-38}$ aspergillosis, ${ }^{9,39-44}$ and paracoccidioidomycosis, ${ }^{45-50}$ the author summarized case reports, as shown in Tables 1-4. Only one case report was published for each of pneumocystosis ${ }^{51}$ and scedosporiosis..$^{52}$

\section{Epidemiology}

Candida spp. are normal commensals of the gastrointestinal tract, ${ }^{53,54}$ whereas Aspergillus spp. and Pneumocystis spp. are ubiquitous in nature ${ }^{5-58}$ and are the cause of fungal infections worldwide. Cryptococcus spp. and Scedosporium spp. are endemic mycoses that also have worldwide distribution. Cryptococcus spp. have been isolated from pigeon droppings, ${ }^{59}$ while Scedosporium spp. have been found in tidal flats, swamps, ponds, manure, and soil. ${ }^{60,61}$ Aspergillus spp. and Cryptococcus spp. can also be found as a component of human colonic mycobiota. ${ }^{62}$

Zygomycetes can be divided into two orders, Mucorales and Entomophthorales. Mucorales are endemic fungi found worldwide in organic substrates, including bread, fruits, vegetable matter, soil, compost, and animal excreta. ${ }^{63}$
Histoplasma spp., Entomophthorales, Paracoccidioides spp., and Penicillium spp. are endemic mycoses that have been isolated from soil in a number of regions of the world. ${ }^{63-71}$ Histoplasma spp. are found in soil enriched with bat, chicken, and blackbird droppings. ${ }^{64,65}$ Entomophthorales can be divided into Conidiobolus spp. and Basidiobolus spp. and are found in the soil of some tropical regions, especially Africa, South America, Central America, and Asia. ${ }^{63}$ Paracoccidioides spp. are limited to soil in Central and South American countries between $23^{\circ}$ north (southern Mexico) and $34.5^{\circ}$ north (Argentina and Uruguay). These areas have unique ecologic features, being tropical and subtropical forests with mild temperatures and high humidity. ${ }^{66,67}$ Penicillium spp. are also isolated from soil in specific areas including Southeast Asia, southern China (Guangxi), Hong Kong, and India. ${ }^{68-71}$ The epidemiologic data is summarized in Table 5.

\section{Prevalence of colonic involvement}

The degree of colonic involvement of fungal infections varies according to fungal type, as detailed in Table 6. Paracoccidioidomycosis and histoplasmosis are the most common colonic infections caused by fungi, with a prevalence of $29 \%{ }^{72}$ and $28 \%,{ }^{20}$ respectively. In contrast, penicilliosis, zygomycosis, pneumocystosis, and scedosporiosis rarely infiltrate the colon, with penicilliosis and zygomycosis occurring in $1.9 \%{ }^{68}$ and $0.85 \%$ of colonic infections, ${ }^{25}$ respectively. Both pneumocystosis and scedosporiosis are known only from one case report. ${ }^{51,52}$ Excluding oropharyngeal candidiasis, the colon is the third most common gastrointestinal organ to be involved in candidiasis following the esophagus and stomach. The colon is involved in $20 \%$ of gastrointestinal candidiasis. ${ }^{73}$ Colonic cryptococcosis is the most common gastrointestinal manifestation of disseminated or pulmonary cryptococcosis, with a prevalence of about $17 \% .{ }^{32}$

\section{Risk factors}

Colonic cryptococcosis and zygomycosis often occur in immunocompromised hosts, occurring in $77 \% \%^{26-35}$ and $67 \%{ }^{22}$ of infections respectively, while colonic candidiasis, ${ }^{13}$ penicilliosis, ${ }^{36-38}$ and aspergillosis ${ }^{9,39-44}$ exclusively occur in immunocompromised hosts. Risk factors for these infections are malignancy, taking immunosuppressive agents, chemotherapy, neutropenia, AIDS, renal failure, splenectomy, Job's syndrome, cirrhosis, malnutrition, and diabetes mellitus; however, most colonic penicilliosis patients usually have AIDS. ${ }^{37,38}$ There have also been case reports of colonic pneumocystosis and scedosporiosis in immunocompromised patients with AIDS and post-liver transplantation, respectively. ${ }^{51,52}$ In colonic paracoccidioidomycosis 


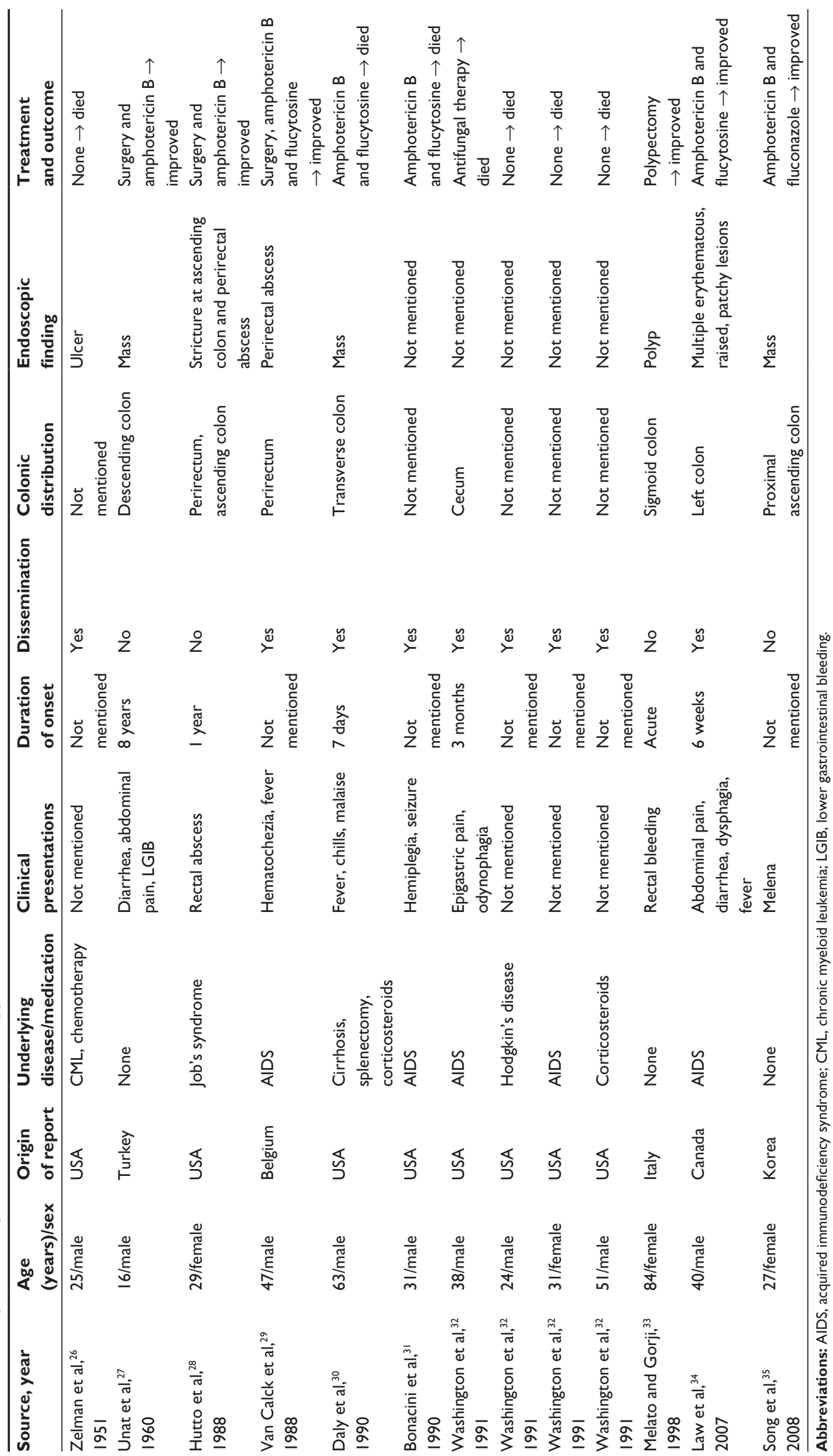




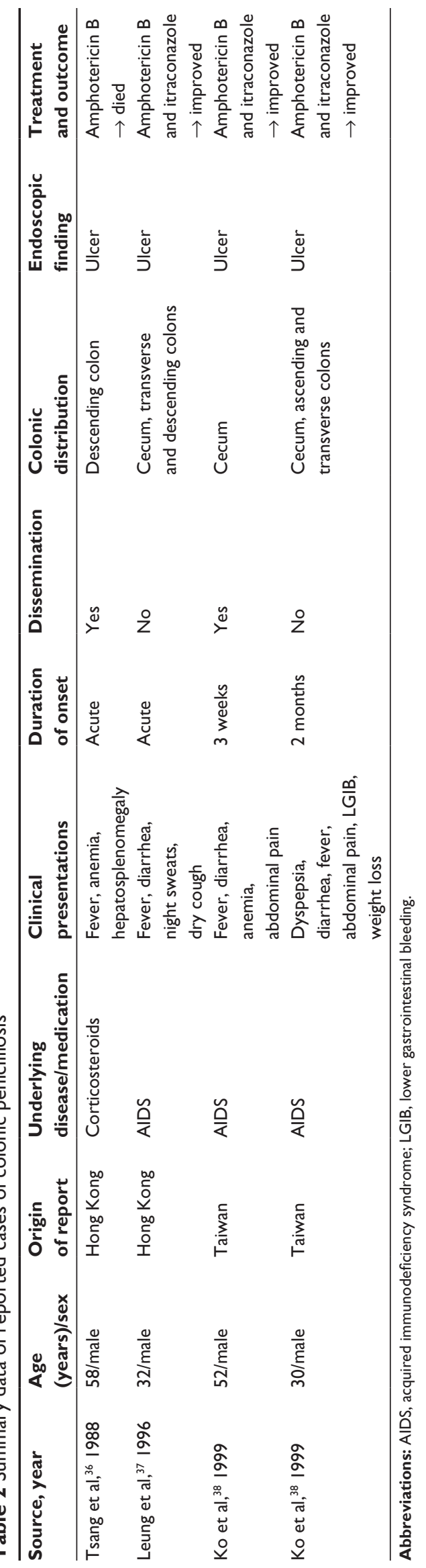

and histoplasmosis, there are no necessary risk factors present. ${ }^{20,21,45-50}$ As shown in Table 7, males predominate in nearly all fungal infections, except for colonic candidiasis, aspergillosis, and paracoccidioidomycosis. The higher frequency of fungal infections in men may be attributed to their more intense exposure to the endemic fungi habitats through work. ${ }^{67}$ There is no sex preference in colonic candidiasis, since Candida spp. are normal commensals of the human gut. ${ }^{13,53,54}$ Aspergillus spp. are ubiquitous, thus sex is also not a risk factor for infection. ${ }^{9,39-44,55,56}$ Although Paracoccidioidomycosis usually occur in men, ${ }^{67}$ females predominate are found in colonic paracoccidioidomycosis. ${ }^{45-50}$ The author suggests that it may be caused by reporting bias.

\section{Immunity and colonic infection caused by fungi}

Host immune response to fungi may play an important role in the pathogenesis of colonic infection caused by fungi, especially organisms that are part of the microbiota. ${ }^{75,76}$ Normally, innate immune cells have membrane-bound and soluble receptors to eliminate fungi. Membrane-bound receptors such as lectin, a toll-like scavenger, and complement receptors can detect fungi or fungal products and then activate phagocytosis and respiratory burst. Transcription factors which can induce proinflammatory cytokines and chemokines are also activated by membrane-bound receptors. Soluble receptors can further activate complements and opsonize fungi to complement receptors. Finally, $\mathrm{T}$ helper (Th) 1 and 17 are triggered and produce other cytokines including interleukin (IL)-17A, IL-17F, and IL-22 for adaptive immunity. Both innate and adaptive immune systems defend the host against fungi. ${ }^{76}$

There is some evidence that impaired immunity can increase colonic infection. IL-22, which is produced by innate cells and regulated by IL-23, has been demonstrated to activate inflammatory cells and thus control initial fungal growth. ${ }^{75}$ Defective IL-23 and IL-22 pathways increase the fungal burden in the gastrointestinal tract; nevertheless, Th1 cells prevent dissemination of fungi. Th17 cells play a major role in adaptive immune responses, though their impairment results in decreased resistance to late fungal infection. IL-17 receptor A deficiency reduces Th1 activation, thus decreasing fungal resistance. ${ }^{75}$ Therefore, immunosuppressive status is one of the major predisposing factors for colonic infection caused by fungi.

\section{Clinical manifestations}

Colonic infections caused by fungi have varied clinical manifestations, as detailed in Table 8. Excluding zygomycosis 


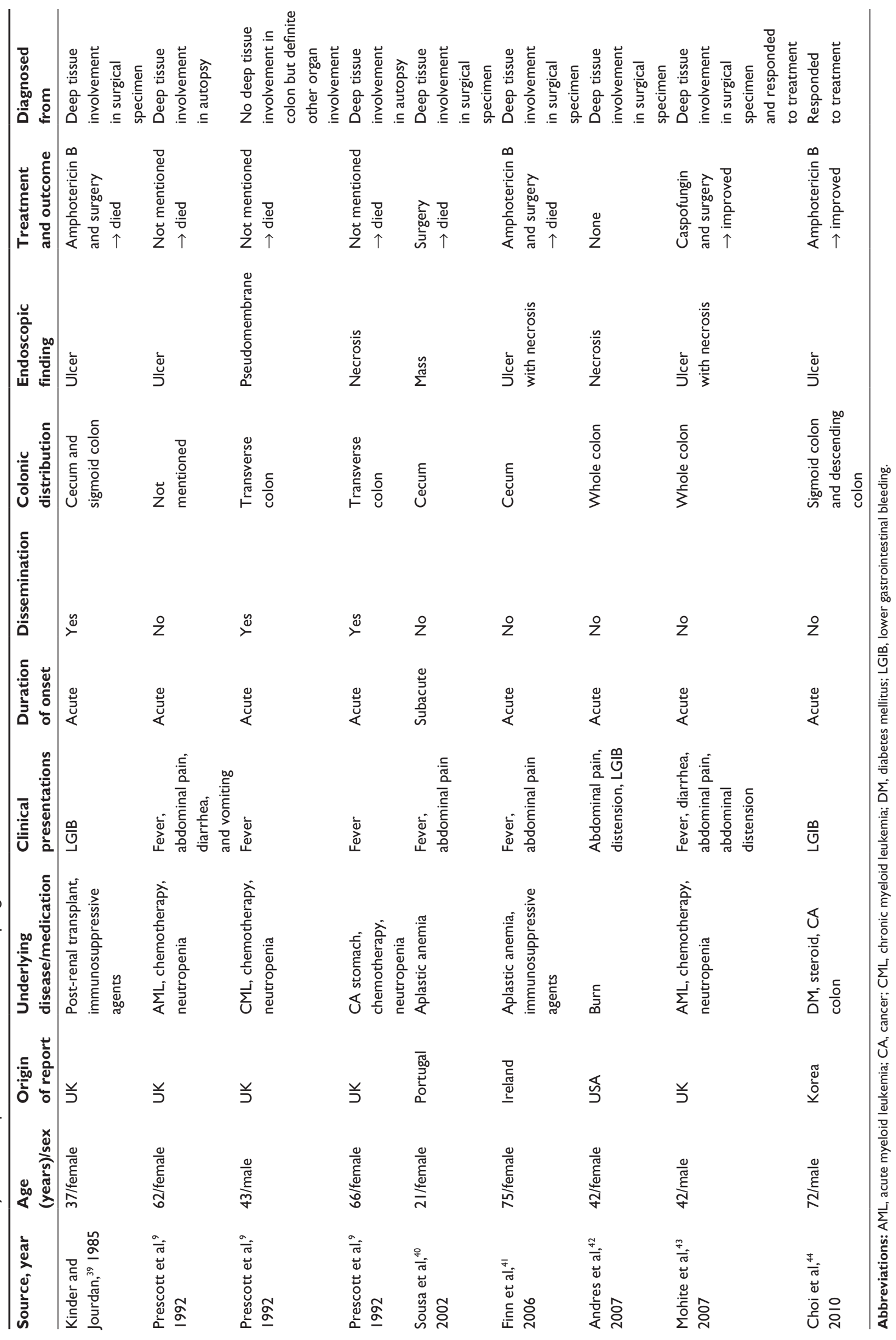


Table 4 Summary data of reported cases of colonic paracoccidioidomycosis

\begin{tabular}{|c|c|c|c|c|c|c|c|c|c|}
\hline $\begin{array}{l}\text { Source, } \\
\text { year }\end{array}$ & $\begin{array}{l}\text { Age(years)/ } \\
\text { sex }\end{array}$ & $\begin{array}{l}\text { Origin } \\
\text { of } \\
\text { report }\end{array}$ & $\begin{array}{l}\text { Underlying } \\
\text { disease/ } \\
\text { medication }\end{array}$ & $\begin{array}{l}\text { Clinical } \\
\text { presentations }\end{array}$ & $\begin{array}{l}\text { Duration } \\
\text { of onset }\end{array}$ & Dissemination & $\begin{array}{l}\text { Colonic } \\
\text { distribution }\end{array}$ & $\begin{array}{l}\text { Endoscopic } \\
\text { finding }\end{array}$ & $\begin{array}{l}\text { Treatment } \\
\text { and outcome }\end{array}$ \\
\hline $\begin{array}{l}\text { Penna, }{ }^{45} \\
1979\end{array}$ & 8/female & Brazil & None & $\begin{array}{l}\text { Diarrhea, abdominal } \\
\text { pain, abdominal } \\
\text { distension, fever, } \\
\text { failure to thrive }\end{array}$ & 4 years & No & Whole colon & $\begin{array}{l}\text { Stricture, } \\
\text { ulcer }\end{array}$ & $\begin{array}{l}\text { Co-trimoxazole } \\
\rightarrow \text { improved }\end{array}$ \\
\hline $\begin{array}{l}\text { Chojniak } \\
\text { et al, }{ }^{46} 2000\end{array}$ & $\begin{array}{l}57 / \text { not } \\
\text { mentioned }\end{array}$ & Brazil & None & $\begin{array}{l}\text { Abdominal pain, } \\
\text { diarrhea, weight loss }\end{array}$ & 2 years & No & Cecum & Mass & $\begin{array}{l}\text { Ketoconazole } \\
\rightarrow \text { not } \\
\text { mentioned }\end{array}$ \\
\hline $\begin{array}{l}\text { Costa Vieira } \\
\text { et al, }{ }^{47} 2001\end{array}$ & $60 /$ male & Brazil & None & $\begin{array}{l}\text { Fever, perianal nodule, } \\
\text { dysphonia, cough, } \\
\text { dyspnea weight loss }\end{array}$ & 2 years & Yes & $\begin{array}{l}\text { Transverse } \\
\text { and } \\
\text { descending } \\
\text { colons }\end{array}$ & Ulcer & $\begin{array}{l}\text { Sulfadiazine } \rightarrow \\
\text { improved }\end{array}$ \\
\hline $\begin{array}{l}\text { Bravo et al, }{ }^{48} \\
2010\end{array}$ & $39 /$ female & Peru & None & $\begin{array}{l}\text { Diarrhea, abdominal } \\
\text { pain, fever, weight loss }\end{array}$ & 2 months & Yes & Whole colon & Ulcer & $\begin{array}{l}\text { Amphotericin B } \\
\rightarrow \text { died }\end{array}$ \\
\hline $\begin{array}{l}\text { Leon et al, } \\
2010\end{array}$ & $34 /$ male & Peru & None & $\begin{array}{l}\text { Diarrhea, oral ucers, } \\
\text { odynophagia, weight } \\
\text { loss, cough }\end{array}$ & 18 months & Yes & Whole colon & Ulcer & $\begin{array}{l}\text { Amphtotericin B } \\
\text { and } \\
\text { itraconazole } \rightarrow \\
\text { improved }\end{array}$ \\
\hline $\begin{array}{l}\text { Leon et al, }{ }^{49} \\
2010\end{array}$ & 40/female & Peru & None & $\begin{array}{l}\text { Diarrhea, weight loss, } \\
\text { hepatomegaly }\end{array}$ & I year & Yes & $\begin{array}{l}\text { Not } \\
\text { mentioned }\end{array}$ & $\begin{array}{l}\text { Not } \\
\text { mentioned }\end{array}$ & $\begin{array}{l}\text { Amphotericin B } \\
\rightarrow \text { died }\end{array}$ \\
\hline $\begin{array}{l}\text { Benard et al, }{ }^{50} \\
2013\end{array}$ & 56/female & Brazil & None & $\begin{array}{l}\text { None (colonoscopy } \\
\text { for check up) }\end{array}$ & Unknown & No & $\begin{array}{l}\text { Transverse } \\
\text { colon }\end{array}$ & Polyp & $\begin{array}{l}\text { Itraconazole } \rightarrow \\
\text { improved }\end{array}$ \\
\hline $\begin{array}{l}\text { Benard et al, }{ }^{50} \\
2013\end{array}$ & $58 /$ female & Brazil & None & $\begin{array}{l}\text { Diarrhea, weight loss, } \\
\text { fever }\end{array}$ & 6 months & No & Whole colon & Ulcer & $\begin{array}{l}\text { Itraconazole } \rightarrow \\
\text { improved }\end{array}$ \\
\hline
\end{tabular}

and aspergillosis, more than one-half of patients with colonic fungal infections have disseminated disease. Abdominal pain is the most common presentation in colonic zygomycosis and aspergillosis..$^{922,24,39-44}$ Deep tissue involvement and angioinvasion, which are common pathological findings in both aspergillosis and zygomycosis, may explain these manifestations. ${ }^{22,25,74}$ Diarrhea is a symptom that is often found in paracoccidioidomycosis and candidiasis. ${ }^{13,45-50}$ Cases of colonic histoplasmosis and penicilliosis include diarrhea and abdominal pain as predominant symptoms..$^{20,21,36-38}$ Perirectal abscess is a specific feature that is only found in colonic cryptococcosis. ${ }^{28,29}$ Fever is a usual finding in fungal infection of the colon, especially in cases of colonic penicilliosis patients, all of whom experience fever. ${ }^{36-38}$ For example, a case of colonic pneumocystosis presented with fever and diarrhea, ${ }^{51}$ while one with colonic scedosporiosis presented with diarrhea and abdominal pain. ${ }^{52}$

\section{Pathological findings and distribution}

Colonic ulcer is the most common pathological finding in patients with fungal infection of the colon, as shown in Table 9. All cases of colonic aspergillosis, and penicilliosis have ulcers. ${ }^{36-38,74}$ Ulcer is also usually found in colonic histoplasmosis, paracoccidioidomycosis, and candidiasis..$^{21,45-50,73}$ Colonic scedosporiosis, likewise, presents with ulcer. ${ }^{52}$ On the contrary, colonic cryptococcosis presents with a mass or polyp as the most common pathologic finding, while colonic cryptococcosis may also present with atypical pathologic findings, including rectal abscess or stricture. ${ }^{26-35}$ Another colonic infection that can present with a mass or polyp is histoplasmosis. ${ }^{21}$ In one case of colonic pneumocystosis, the patient presented with bowel edema. ${ }^{51}$

Distributions of colonic infection caused by fungi differ according to fungus type, as shown in Table 10. Colonic histoplasmosis, zygomycosis, and penicilliosis tend to occur

Table 5 Epidemiology of fungi that can cause colonic infection

\begin{tabular}{ll}
\hline Fungi & Epidemiology \\
\hline Aspergillus spp., ${ }^{55,56}$ Candida spp., ${ }^{53,54}$ Cryptococcus spp., ${ }^{59}$ Histoplasma spp., \\
Mucorales ${ }^{63}$ Pneumocystis spp., $^{57,58}$ Scedosporium spp. ${ }^{60,61}$ & Worldwide \\
Entomophthorales $^{63}$ & Tropical areas in Africa, South America, Central America, and Asia \\
Paracoccidioides spp. ${ }^{66,67}$ & South and Central American countries, particularly in Brazil, Colombia, \\
Penicillium spp. ${ }^{68-71}$ & Venezuela, and Argentina \\
\hline
\end{tabular}


Table 6 Prevalence of colonic involvement in each fungal infection

\begin{tabular}{lll}
\hline Fungal infections & Prevalence of colonic involvement & Comments \\
\hline Paracoccidioidomycosis $^{72}$ & $29 \%$ & Prevalence ascertained by autopsy series \\
Histoplasmosis $^{20}$ & $28 \%$ & \\
Candidiasis $^{73}$ & $20 \%$ of gastrointestinal candidiasis (excluding & Prevalence ascertained by autopsy series \\
& oropharyngeal candidiasis) & Only seven symptomatic cases \\
Cryptococcosis $^{32}$ & $17 \%$ of disseminated or pulmonary cryptococcosis & \\
Aspergillosis $^{74}$ & $9.2 \%$ & Only four cases \\
Penicilliosis $^{68}$ & $1.9 \%$ & \\
Zygomycosis $^{25}$ & $0.85 \%$ & Only one case \\
Pneumocystosis & No data & Only one case \\
\hline Scedosporiosis & & No data
\end{tabular}

Table 7 Risk factors for fungal infections of the colon

\begin{tabular}{|c|c|c|c|c|c|}
\hline Fungal infections & Immunocompetent & Immunocompromised & Risk factors & Male & Comments \\
\hline Paracoccidioidomycosis ${ }^{45-50}$ & $100 \%$ & $0 \%$ & - & $29 \%$ & \\
\hline Histoplasmosis $^{20,21}$ & $81 \%$ & $19 \%$ & $\begin{array}{l}\text { Malignancy, } \\
\text { immunosuppressive drugs, } \\
\text { AIDS, Job's syndrome, } \\
\text { DM, splenectomy }\end{array}$ & $76 \%-86.5 \%$ & \\
\hline Candidiasis $^{13}$ & $0 \%$ & $100 \%$ & $\begin{array}{l}\text { Malignancy, } \\
\text { immunosuppressive agents, } \\
\text { neutropenia, AIDS, ESRD }\end{array}$ & $43 \%$ & \\
\hline Cryptococcosis ${ }^{26-35}$ & $23 \%$ & $77 \%$ & $\begin{array}{l}\text { AIDS, immunosuppressive } \\
\text { agents, hematologic } \\
\text { malignancy, splenectomy, } \\
\text { Job's syndrome, cirrhosis }\end{array}$ & $64 \%$ & \\
\hline Aspergillosis i, $^{99-44}$ & $0 \%$ & $100 \%$ & $\begin{array}{l}\text { Malignancy, chemotherapy, } \\
\text { neutropenia, } \\
\text { immunosuppressive } \\
\text { agents, DM, burn }\end{array}$ & $33 \%$ & \\
\hline Penicilliosis ${ }^{36-38}$ & $0 \%$ & $100 \%$ & $\begin{array}{l}\text { AIDS (75\%), } \\
\text { immunosuppressive agents }\end{array}$ & $100 \%$ & \\
\hline Zygomycosis ${ }^{22,24}$ & $33 \%$ & $67 \%$ & $\begin{array}{l}\text { Immunosuppressive } \\
\text { agents, malnutrition, } \\
\text { renal failure, DM, } \\
\text { hematologic malignancy }\end{array}$ & $65 \%$ & \\
\hline Pneumocystosis ${ }^{51}$ & $0 \%$ & $100 \%$ & AIDS & $100 \%$ & $\begin{array}{l}\text { Only one } \\
\text { case }\end{array}$ \\
\hline Scedosporiosis ${ }^{52}$ & $0 \%$ & $100 \%$ & $\begin{array}{l}\text { Post-liver transplantation, } \\
\text { immunosuppressive agents }\end{array}$ & $100 \%$ & $\begin{array}{l}\text { Only one } \\
\text { case }\end{array}$ \\
\hline
\end{tabular}

Abbreviations: AIDS, acquired immunodeficiency syndrome; DM, diabetes mellitus; ESRD, end-stage renal disease.

Table 8 Clinical manifestations of colonic infections caused by fungi

\begin{tabular}{|c|c|c|c|c|c|c|c|}
\hline Fungal infections & Dissemination & Diarrhea & $\begin{array}{l}\text { Abdominal } \\
\text { pain }\end{array}$ & LGIB & $\begin{array}{l}\text { Rectal } \\
\text { abscess }\end{array}$ & Fever & Comments \\
\hline Paracoccidioidomycosis ${ }^{45-50}$ & $50 \%$ & $75 \%$ & $38 \%$ & - & - & $50 \%$ & $\begin{array}{l}\text { Asymptomatic in } \\
12.5 \%\end{array}$ \\
\hline Histoplasmosis ${ }^{20,21}$ & $83 \%$ & $83 \%$ & $67 \%$ & $32 \%$ & - & $77 \%$ & - \\
\hline Candidiasis ${ }^{13}$ & $71 \%$ & $57 \%$ & $29 \%$ & $29 \%$ & - & $71 \%$ & - \\
\hline Cryptococcosis ${ }^{26-35}$ & $\begin{array}{l}\text { 71\% (all patients } \\
\text { immunocompromised) }\end{array}$ & $20 \%$ & $30 \%$ & $40 \%$ & $20 \%$ & $30 \%$ & Asymptomatic in $20 \%$ \\
\hline Aspergillosis ${ }^{9,39-44}$ & $33 \%$ & $22 \%$ & $56 \%$ & $33 \%$ & - & $67 \%$ & - \\
\hline Penicilliosis ${ }^{36-38}$ & $50 \%$ & $75 \%$ & $75 \%$ & $25 \%$ & - & $100 \%$ & - \\
\hline Zygomycosis ${ }^{22,24}$ & $38 \%$ & $18 \%$ & $64 \%$ & $18 \%$ & - & $55 \%$ & - \\
\hline Pneumocystosis ${ }^{51}$ & $100 \%$ & $100 \%$ & - & - & - & $100 \%$ & Only one case \\
\hline Scedosporiosis ${ }^{52}$ & $100 \%$ & $100 \%$ & $100 \%$ & - & - & - & Only one case \\
\hline
\end{tabular}

Note: Dissemination is defined as involvement of noncontiguous organs. ${ }^{15-19}$

Abbreviation: LGIB, lower gastrointestinal bleeding. 
Table 9 Pathological findings of colonic infections caused by fungi

\begin{tabular}{|c|c|c|c|c|c|c|c|}
\hline Fungal infections & Ulcer & $\begin{array}{l}\text { Inflamed } \\
\text { mucosa/erosion }\end{array}$ & Pseudomembrane & $\begin{array}{l}\text { Mass/ } \\
\text { polyp }\end{array}$ & $\begin{array}{l}\text { Rectal } \\
\text { abscess }\end{array}$ & Stricture & Comment \\
\hline Paracoccidioidomycosis ${ }^{45-50}$ & $63 \%$ & - & - & $25 \%$ & - & $13 \%$ & \\
\hline Histoplasmosis ${ }^{21}$ & $79 \%$ & $14 \%$ & - & $7 \%$ & - & - & \\
\hline Candidiasis $^{73}$ & $64 \%$ & $14 \%$ & $23 \%$ & - & - & - & $\begin{array}{l}\text { From autopsy } \\
\text { series }\end{array}$ \\
\hline Cryptococcosis $26-35$ & $11 \%$ & $11 \%$ & - & $44 \%$ & $22 \%$ & $11 \%$ & \\
\hline Aspergillosis ${ }^{74}$ & $\begin{array}{l}100 \% \text { (with necrosis } \\
55.6 \% \text { ) }\end{array}$ & - & - & - & - & - & $\begin{array}{l}\text { From autopsy } \\
\text { series }\end{array}$ \\
\hline Penicilliosis ${ }^{36-38}$ & $100 \%$ & - & - & - & - & - & \\
\hline Zygomycosis s2,25 $^{20}$ & - & - & - & - & - & - & $\begin{array}{l}\text { Ulcer, } \\
\text { necrosis, mass } \\
\text { occured, but } \\
\text { numbers of } \\
\text { patients not } \\
\text { specified }\end{array}$ \\
\hline Pneumocystosis ${ }^{51}$ & - & $100 \%$ & - & - & - & - & Only one case \\
\hline Scedosporiosis ${ }^{52}$ & $100 \%$ & - & - & - & - & - & Only one case \\
\hline
\end{tabular}

Table 10 Distributions of colonic infections caused by fungi

\begin{tabular}{|c|c|c|c|c|c|c|c|}
\hline Fungal infections & $\begin{array}{l}\text { Cecum or ascending } \\
\text { colon or appendix }\end{array}$ & $\begin{array}{l}\text { Transverse } \\
\text { colon }\end{array}$ & $\begin{array}{l}\text { Descending } \\
\text { or sigmoid colon }\end{array}$ & Rectum & Perirectum & $\begin{array}{l}\text { Whole } \\
\text { colon }\end{array}$ & Comments \\
\hline Paracoccidioidomycosis ${ }^{45-50}$ & $13 \%$ & $25 \%$ & $13 \%$ & - & - & $50 \%$ & \\
\hline Histoplasmosis ${ }^{20}$ & $66 \%$ & - & $8 \%$ & $26 \%$ & - & - & \\
\hline Candidiasis $^{13}$ & $20 \%$ & - & $40 \%$ & $20 \%$ & - & $20 \%$ & \\
\hline Cryptococcosis is-30,32-35 $^{27}$ & $30 \%$ & $10 \%$ & $40 \%$ & - & $20 \%$ & - & \\
\hline Aspergillosis ${ }^{9,39-44}$ & $33 \%$ & $22 \%$ & $22 \%$ & - & - & $22 \%$ & \\
\hline Penicilliosis ${ }^{36-38}$ & $50 \%$ & $25 \%$ & $25 \%$ & - & - & - & \\
\hline Zygomycosis ${ }^{23}$ & $50 \%$ & - & $18 \%$ & $7 \%$ & - & $25 \%$ & \\
\hline Pneumocystosis ${ }^{51}$ & - & - & - & - & - & $100 \%$ & $\begin{array}{l}\text { Only one } \\
\text { case }\end{array}$ \\
\hline Scedosporiosis ${ }^{52}$ & - & - & - & - & - & $100 \%$ & $\begin{array}{l}\text { Only one } \\
\text { case }\end{array}$ \\
\hline
\end{tabular}

Table I I Treatment response of fungal infections in the colon

\begin{tabular}{|c|c|c|c|}
\hline Fungal infections & Treatment & $\begin{array}{l}\text { Treatment } \\
\text { response }\end{array}$ & Comments \\
\hline Paracoccidioidomycosis ${ }^{45,47-50}$ & $\begin{array}{l}\text { Co-trimoxazole, sulfadiazine, amphotericin B, } \\
\text { or itraconazole }\end{array}$ & $71 \%$ & \\
\hline Histoplasmosis ${ }^{21}$ & Amphotericin B & $77 \%$ & \\
\hline Candidiasis $^{13}$ & Fluconazole or caspofungin & $100 \%$ & \\
\hline Cryptococcosis is-35 $^{27}$ & Amphotericin B + flucytosine \pm surgery & $67 \%$ & $\begin{array}{l}\text { Response to treatment in } \\
\text { immunocompetent patients was } 100 \%\end{array}$ \\
\hline Aspergillosis ${ }^{39,41,43,44}$ & Amphotericin B or caspofungin \pm surgery & $50 \%$ & \\
\hline Penicilliosis ${ }^{36-38}$ & Amphotericin B & $75 \%$ & \\
\hline Zygomycosis ${ }^{25}$ & Amphotericin $B+$ surgery & $50 \%$ & $\begin{array}{l}\text { Combined surgery improved } \\
\text { treatment response }\end{array}$ \\
\hline Pneumocystosis $^{51}$ & Pentamidine & $100 \%$ & Only one case \\
\hline Scedosporiosis ${ }^{52}$ & Amphotericin B & $100 \%$ & Only one case \\
\hline
\end{tabular}




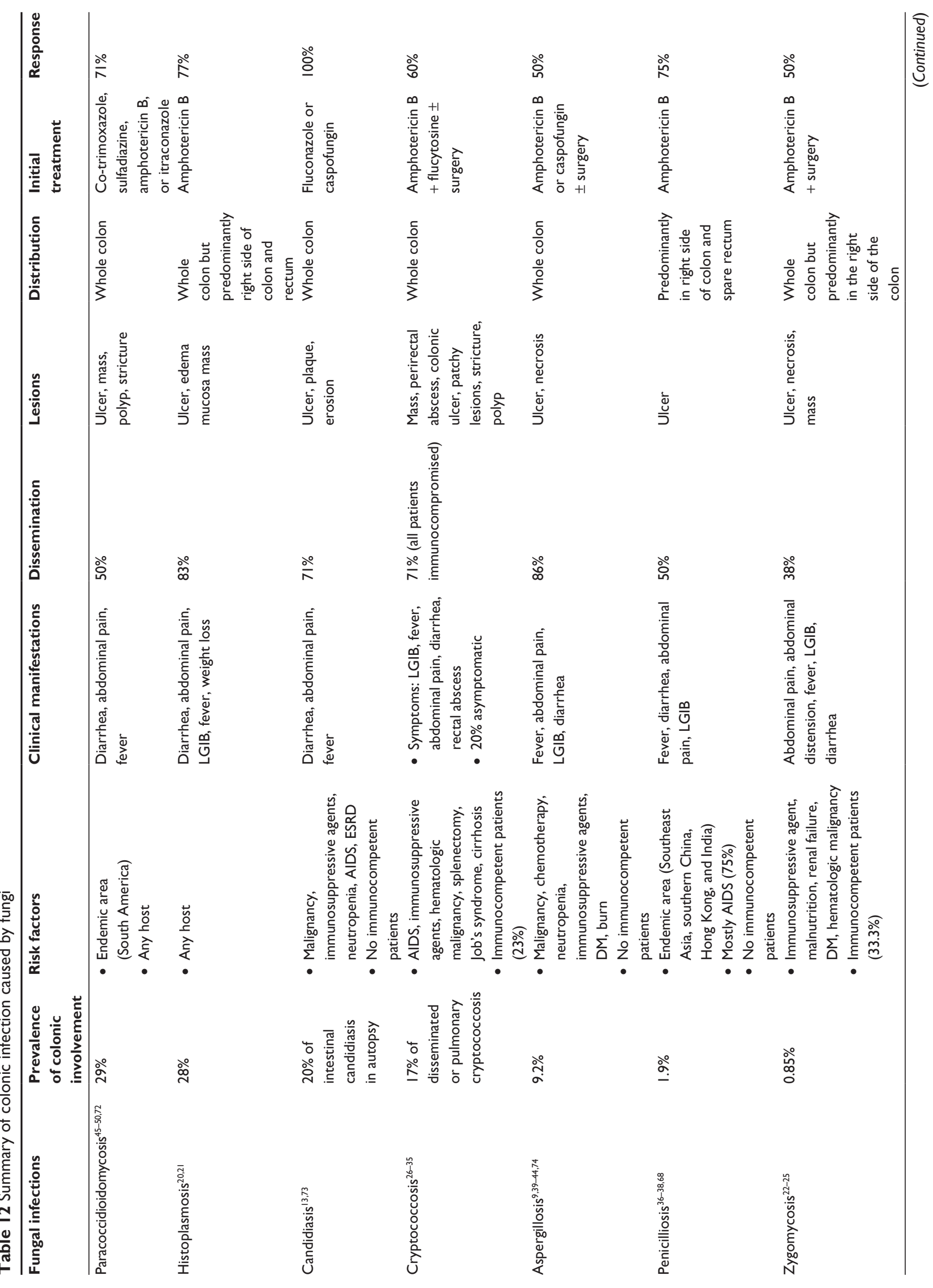


in the right side of the colon. ${ }^{20,23,36-38}$ The rectal area tends to be involved in cases of histoplasmosis, candidiasis, and zygomycosis. ${ }^{13,20,23}$ Colonic cryptococcosis is the only fungus that involves the perirectal area. ${ }^{28,29}$ Diffuse involvement of the colon is commonly found in colonic paracoccidioidomycosis. ${ }^{45-50}$ Cases of both colonic pneumocystosis and scedosporiosis also presented with diffuse lesions. ${ }^{51,52}$

\section{Treatment response}

Amphotericin B is the most commonly used drug to treat nearly all colonic fungal infections except candidiasis and pneumocystosis. Colonic candidiasis responds to fluconazole or caspofungin and has the best prognosis if antifungal therapy is initiated with $100 \%$ compliance. ${ }^{13}$ Good response was also achieved in colonic pneumocystosis and scedosporiosis. . $^{51,52}$ A case of colonic pneumocystosis was treated with intravenous pentamidine due to sulfamethoxazole-trimethoprim allergy. ${ }^{51}$ Combined antifungal therapy and surgery have been used to treat gastrointestinal zygomycosis and aspergillosis due to angioinvasion and infarction. Nevertheless, prognosis is still poor in these cases, with $50 \%$ mortality. ${ }^{25,39,41,43,44} \mathrm{The}$ choices of antibiotic and treatment response of fungal infections are summarized in Table 11.

\section{Conclusion}

This is the first study to intensively review the literature on fungal infections of the colon. The entire content is summarized in Table 12. It provides basic information on causes, manifestations, and management and can be easily applied in clinical practice. Physicians should be aware of this fungal entity when patients have colonic symptoms, especially in immunocompromised cases. Although many fungal infections have been reported to cause colonic disease, including aspergillosis, candidiasis, cryptococcosis, histoplasmosis, paracoccidioidomycosis, penicilliosis, pneumocystosis, scedosporiosis, and zygomycosis, knowing the differences in epidemiology, risk factors, clinical manifestations, and pathological findings will help physicians to better diagnosis and manage these infections. Appropriate treatment with antifungal therapy definitely improves outcomes; nevertheless, zygomycosis and aspergillosis cases still have high mortality rates.

\section{Disclosure}

The author reports no conflicts of interest in this work.

\section{References}

1. Anaissie EJ, McGinnis MR, Pfaller MA, editors. Clinical Mycology. New York, NY: Churchill Livingstone; 2003. 
2. Dismukes WE, Pappas PG, Sobel JD, editors. Clinical Mycology. New York, NY: Oxford University Press; 2003.

3. Hospenthal DR, Rinaldi MG, editors. Diagnosis and Treatment of Human Mycoses. Totowa, NJ: Humana Press; 2008.

4. Smith JM. Mycoses of the alimentary tract. Gut. 1969;10:1035-1040.

5. Schmitt SL, Wexner SD. Bacterial, fungal, parasitic, and viral colitis. Surg Clin North Am. 1993;73(5):1055-1062.

6. Lima AAM, Guerrant RL. Inflammatory enteritides. In: Mandell GL, Bennett JE, Dolin R, editors. Mandell, Douglas, and Bennett's Principles and Practice of Infectious Diseases. 7th ed. Philadelphia, PA: Churchill Livingstone Elsevier; 2009:1389-1398.

7. Wilcox CM. Gastrointestinal consequences of infection with human immunodeficiency virus. In: Feldman M, Friedman LS, Brandt LJ, editors. Sleisenger and Fordtran's Gastrointestinal and Liver Disease. 9th ed. Philadelphia, PA: Saunders Elsevier; 2010:523-535.

8. Stylianos S, Forde KA, Benvenisty Al, Hardy MA. Lower gastrointestinal hemorrhage in renal transplant recipients. Arch Surg. 1988;123:739-744.

9. Prescott RJ, Harris M, Banerjee SS. Fungal infections of the small and large intestine. J Clin Pathol. 1992;45:806-811.

10. Jayagopal S, Cervia JS. Colitis due to Candida albicans in a patient with AIDS. Clin Infect Dis. 1992;15:555.

11. Kouklakis G, Dokas S, Molyvas E, Vakianis P, Efthymiou A. Candida colitis in a middle-aged male receiving permanent haemodialysis. Eur J Gastroenterol Hepatol. 2001;13:735-736.

12. Kitagawa KH, Kalb RE. Efalizumab treatment associated with Candida colitis. J Am Acad Dermatol. 2008;59(suppl 5):S120-S121.

13. Praneenararat $\mathrm{S}$. The first reported case of colonic infection caused by Candida tropicalis and a review of the literature. Case Rep Gastroenterol. 2014;8:199-205.

14. De Pauw B, Walsh TJ, Donnelly JP, et al. Revised definitions of invasive fungal disease from the European Organization for Research and Treatment of Cancer/Invasive Fungal Infections Cooperative Group and the National Institute of Allergy and Infectious Diseases Mycoses Study Group (EORTC/ MSG) Consensus Group. Clin Infect Dis. 2008;46:1813-1821.

15. Ascioglu S, Rex JH, de Pauw B, et al; Invasive Fungal Infections Cooperative Group of the European Organization for Research and Treatment of Cancer; Mycoses Study Group of the National Institute of Allergy and Infectious Diseases. Defining opportunistic invasive fungal infections in immunocompromised patients with cancer and hematopoietic stem cell transplants: an international consensus. Clin Infect Dis. 2002;34:7-14.

16. Safdar A, Singhal S, Mehta J. Clinical significance of non-Candida fungal blood isolation in patients undergoing high-risk allogeneic hematopoietic stem cell transplantation (1993-2001). Cancer. 2004;100:2456-2461.

17. Kontoyiannis DP, Bodey GP, Hanna H, et al. Outcome determinants of fusariosis in a tertiary care cancer center: the impact of neutrophil recovery. Leuk Lymphoma. 2004;45:139-141.

18. Lionakis MS, Bodey GP, Tarrand JJ, Raad II, Kontoyiannis DP. The significance of blood cultures positive for emerging saprophytic moulds in cancer patients. Clin Microbiol Infect. 2004;10:922-925.

19. Safdar A, Rodriguez G, Rolston KV, et al. High-dose caspofungin combination antifungal therapy in patients with hematologic malignancies and hematopoietic stem cell transplantation. Bone Marrow Transplant. 2007;39(3):157-164.

20. Cappell MS, Mandell W, Grimes MM, Neu HC. Gastrointestinal histoplasmosis. Dig Dis Sci. 1988;33:353-360.

21. Assi M, McKinsey DS, Driks MR, et al. Gastrointestinal histoplasmosis in the acquired immunodeficiency syndrome: report of 18 cases and literature review. Diagn Microbiol Infect Dis. 2006;55:195-201.

22. Lyon DT, Schubert TT, Mantia AG, Kaplan MH. Phycomycosis of the gastrointestinal tract. Am J Gastroenterol. 1979;72(4):379-394.

23. Agha FP, Lee HH, Boland CR, Bradley SF. Mucormycoma of the colon: early diagnosis and successful management. AJR Am J Roentgenol. 1985;145:739-741.

24. Roden MM, Zaoutis TE, Buchanan WL, et al. Epidemiology and outcome of zygomycosis: a review of 929 reported cases. Clin Infect Dis. 2005;41:634-653.
25. Almyroudis NG, Sutton DA, Linden P, Rinaldi MG, Fung J, Kusne S. Zygomycosis in solid organ transplant recipients in a tertiary transplant center and review of the literature. Am J Transplant. 2006;6: 2365-2374.

26. Zelman S, O'Neil RH, Plaut A. Disseminated visceral torulosis without nervous system involvement with clinical appearance of granulocytic leukemia. Am J Med. 1951;11:658-664.

27. Unat EK, Pars B, Kosyak JP. A case of cryptococcosis of the colon. Br Med J. 1960;2:1501-1502.

28. Hutto JO, Bryan CS, Greene FL, White CJ, Gallin JI. Cryptococcosis of the colon resembling Crohn's disease in a patient with the hyperimmunoglobulinemia E recurrent infection (Job's) syndrome. Gastroenterology. 1988;94:808-812.

29. Van Calck M, Motte S, Rickaert F, Serruys E, Adler M, Wybran J. Cryptococcal anal ulceration in a patient with AIDS. Am J Gastroenterol. 1988;83:1306-1308.

30. Daly JS, Porter KA, Chong FK, Robillard RJ. Disseminated, nonmeningeal gastrointestinal cryptococcal infection in an HIV-negative patient. Am J Gastroenterol. 1990;85:1421-1424.

31. Bonacini M, Nussbaum J, Ahluwalia C. Gastrointestinal, hepatic, and pancreatic involvement with Cryptococcus neoformans in AIDS. J Clin Gastroenterol. 1990;12:295-297.

32. Washington K, Gottfried MR, Wilson ML. Gastrointestinal cryptococcosis. Mod Pathol. 1991;4:707-711.

33. Melato M, Gorji N. Primary intestinal cryptococcosis mimicking adenomatous polyp in an HIV-negative patient. Am J Gastroenterol. 1998;93:1592-1593.

34. Law JK, Amar JN, Kirby SD, Zetler PJ, Enns RA. Colonic cryptococcus infection. Gastrointest Endosc. 2007;65:525-526.

35. Song JC, Kim SK, Kim ES, et al. [A case of colonic cryptococcosis]. Korean J Gastroenterol. 2008;52:255-260. Korean.

36. Tsang DN, Chan JK, Lau YT, Lim W, Tse CH, Chan NK. Penicillium marneffei infection: an underdiagnosed disease? Histopathology. 1988; 13:311-318.

37. Leung R, Sung JY, Chow J, Lai CK. Unusual cause of fever and diarrhea in a patient with AIDS. Penicillium marneffei infection. Dig Dis Sci. 1996;41:1212-1215.

38. Ko CI, Hung CC, Chen MY, Hsueh PR, Hsiao CH, Wong JM. Endoscopic diagnosis of intestinal penicilliosis marneffei: report of three cases and review of the literature. Gastrointest Endosc. 1999;50(1): 111-114.

39. Kinder RB, Jourdan MH. Disseminated aspergillosis and bleeding colonic ulcers in renal transplant patient. $J R$ Soc Med. 1985;78(4):338-339.

40. Sousa AB, Ferreira G, Veiga J, Carvalho A. Clinical picture: bowel infarction due to aspergillosis. Lancet. 2002;359(9302):210.

41. Finn S, Bond J, McCarthy D, Sheahan K, Quinn C. Angioinvasive aspergillosis presenting as neutropenic colitis. Histopathology. 2006;49(4):440-441.

42. Andres LA, Ford RD, Wilcox RM. Necrotizing colitis caused by systemic aspergillosis in a burn patient. J Burn Care Res. 2007;28(6): 918-921.

43. Mohite U, Kell J, Haj MA, et al. Invasive aspergillosis localised to the colon presenting as toxic megacolon. Eur J Haematol. 2007;78: 270-273.

44. Choi SH, Chung JW, Cho SY, Kim BJ, Kwon GY. A case of isolated invasive Aspergillus colitis presenting with hematochezia in a nonneutropenic patient with colon cancer. Gut Liver. 2010;4(2):274-277.

45. Penna FJ. Blastomycosis of the colon resembling ulcerative colitis. Gut. 1979;20:896-899.

46. Chojniak R, Costa Vieira RA, Lopes A, et al. Intestinal paracoccidioidomycosis simulating colon cancer. Rev Soc Bras Med Trop. 2000;33:309-312.

47. Costa Vieira RA, Lopes A, Oliveira HV, et al. Anal paracoccidioidomycosis: an unusual presentation of disseminated disease. Rev Soc Med Trop. 2001;34:583-586.

48. Bravo EA, Zegarra AJ, Piscoya A, et al. Chronic diarrhea and pancolitis caused by paracoccidioidomycosis: case report. Case Report Med. 2010;2010:140505. 
49. León M, Alave J, Bustamante B, Cok J, Gotuzzo E, Seas C. Human T lymphotropic virus 1 and paracoccidioidomycosis: a probable association in Latin America. Clin Infect Dis. 2010;51(2):250-251.

50. Benard G, Costa AN, Leopércio AP, Vicentini AP, Kono A, ShikanaiYasuda MA. Chronic paracoccidioidomycosis of the intestine as single organ involvement points to an alternative pathogenesis of the mycosis. Mycopathologia. 2013;176(5-6):353-357.

51. Bellomo AR, Perlman DC, Kaminsky DL, Brettholz EM, Sarlin JG. Pneumocystis colitis in a patient with the acquired immunodeficiency syndrome. Am J Gastroenterol. 1992;87(6):759-761.

52. Lin D, Kamili Q, Lai S, Musher DM, Hamill R. Cerebral Scedosporium apiospermum infection presenting with intestinal manifestations. Infection. 2013;41(3):723-726.

53. Gorbach SL, Nahas L, Lerner PI, Weinstein L. Studies of intestinal microflora. I. Effects of diet, age, and periodic sampling on numbers of fecal microorganisms in man. Gastroenterology. 1967;53:845-855.

54. Cohen R, Roth FJ, Delgado E, Ahearn DG, Kalser MH. Fungal flora of the normal human small and large intestine. N Engl J Med. 1969;280: 638-641.

55. Denning DW. Invasive aspergillosis. Clin Infect Dis. 1998;26: 781-803.

56. Marr KA, Patterson T, Denning D. Aspergillosis. Pathogenesis, clinical manifestations, and therapy. Infect Dis Clin North Am. 2002;16: 875-894, vi.

57. Smulian AG, Keely SP, Sunkin SM, Stringer JR. Genetic and antigenic variation in Pneumocystis carinii organisms: tools for examining the epidemiology and pathogenesis of infection. J Lab Clin Med. 1997;130(5):461-468.

58. Daly KR, Koch J, Levin L, Walzer PD. Enzyme-linked immunosorbent assay and serologic responses to Pneumocystis jiroveci. Emerg Infect Dis. 2004;10(5):848-854.

59. Chayakulkeeree M, Perfect JR. Cryptococcosis. Infect Dis Clin North Am. 2006;20:507-544.

60. Salkin IF, McGinnis MR, Dykstra MJ, Rinaldi MG. Scedosporium inflatum, an emerging pathogen. J Clin Microbiol. 1988;26: 498-503.

61. Summerbell RC, Krajden S, Kane J. Potted plants in hospitals as reservoirs of pathogenic fungi. Mycopathologia. 1989;106:13-22.

62. Hoffmann C, Dollive S, Grunberg S, et al. Archaea and fungi of the human gut microbiome: correlations with diet and bacterial residents. PLoS One. 2013;8(6):e66019.
63. Kontoyiannis DP, Lewis RE. Invasive zygomycosis: update on pathogenesis, clinical manifestations, and management. Infect Dis Clin North Am. 2006;20:581-607.

64. Goodwin RA Jr, Shapiro JL, Thurman GH, Thurman SS, Des Prez RM. Disseminated histoplasmosis: clinical and pathologic correlations. Medicine (Baltimore). 1980;59:1-33.

65. Wheat LJ, Slama TG, Eitzen HE, Kohler RB, French ML, Biesecker JL. A large urban outbreak of histoplasmosis: clinical features. Ann Intern Med. 1981;94:331-337.

66. Negroni R. Paracoccidioidomycosis (South American blastomycosis, Lutz's mycosis). Int J Dermatol. 1993;32:847-859.

67. Blotta MH, Mamoni RL, Oliveira SJ, et al. Endemic regions of paracoccidioidomycosis in Brazil: a clinical and epidemiologic study of 584 cases in the southeast region. Am J Trop Med Hyg. 1999;61: 390-394.

68. Supparatpinyo K, Khamwan C, Baosoung V, Nelson KE, Sirisanthana T. Disseminated Penicillium marneffei infection in southeast Asia. Lancet. 1994;344:110-113.

69. Wong KH, Lee SS, Lo YC, et al. Profile of opportunistic infections among HIV-1 infected people in Hong Kong. Zhonghua Yi Xue Za Zhi (Taipei). 1995;55:127-136.

70. Duong TA. Infection due to Penicillium marneffei, an emerging pathogen: review of 155 reported cases. Clin Infect Dis. 1996;23:125-130.

71. Wong KH, Lee SS. Comparing the first and second hundred AIDS cases in Hong Kong. Singapore Med J. 1998;39:236-240.

72. Peña CE. Deep mycotic infections in Colombia. A clinicopathologic study of 162 cases. Am J Clin Pathol. 1967;47(4):505-520.

73. Eras P, Goldstein MJ, Sherlock P. Candida infection of the gastrointestinal tract. Medicine (Baltimore). 1972;51:367-379.

74. Young RC, Bennett JE, Vogel CL, Carbone PP, DeVita VT. Aspergillosis. The spectrum of the disease in 98 patients. Medicine (Baltimore). 1970;49:147-173.

75. De Luca A, Zelante T, D'Angelo C, et al. IL-22 defines a novel immune pathway of antifungal resistance. Mucosal Immunol. 2010;3(4): 361-373.

76. Underhill DM, Iliev ID. The mycobiota: interactions between commensal fungi and the host immune system. Nat Rev Immunol. 2014;14(6): 405-416.
Clinical and Experimental Gastroenterology

\section{Publish your work in this journal}

Clinical and Experimental Gastroenterology is an international, peerreviewed, open access journal, publishing all aspects of gastroenterology in the clinic and laboratory, including: Pathology, pathophysiology of gastrointestinal disease; Investigation and treatment of gastointestinal disease; Pharmacology of drugs used in the alimentary tract;

\section{Dovepress}

Immunology/genetics/genomics related to gastrointestinal disease. This journal is indexed on CAS. The manuscript management system is completely online and includes a very quick and fair peer-review system. Visit http://www.dovepress.com/testimonials.php to read real quotes from published authors. 